\title{
Energy Efficiency Analysis of Cache-Enabled Cellular Networks with Limited Backhaul
}

\author{
Congshan Fan, ${ }^{1}$ Tiankui Zhang $\mathbb{D}^{1},{ }^{1}$ Zhimin Zeng, ${ }^{1}$ and Yue Chen ${ }^{2}$ \\ ${ }^{1}$ Beijing Key Laboratory of Network System Architecture and Convergence, School of Information and Communication Engineering, \\ Beijing University of Posts and Telecommunications, Beijing, China \\ ${ }^{2}$ School of Electronic Engineering and Computer Science, Queen Mary University of London, London, UK \\ Correspondence should be addressed to Tiankui Zhang; tkzhang@gmail.com
}

Received 15 December 2017; Revised 15 January 2018; Accepted 18 January 2018; Published 19 February 2018

Academic Editor: Lisheng Fan

Copyright (c) 2018 Congshan Fan et al. This is an open access article distributed under the Creative Commons Attribution License, which permits unrestricted use, distribution, and reproduction in any medium, provided the original work is properly cited.

Caching in the cellular networks has been proposed as a promising technology for reducing the content delivery latency and backhaul cost. Since the backhaul capacity is limited in the practical scenario, the network performance analysis of base station (BS) caching should address the effects of the limited backhaul. This paper investigates the energy efficiency of the cache-enabled cellular networks with the limited backhaul based on the stochastic geometry method. First, the successful content delivery probability (SCDP), which depends on the successful access delivery probability, successful backhaul delivery probability, and cache hit ratio, is analyzed under the limited backhaul. Based on the obtained SCDP results, we derive the analytical expressions of throughput, power consumption, and energy efficiency for various scenes including the general case, the interference-limited case, and the mean load approximation case. The accuracy of theoretical analysis is verified by the Monte Carlo simulation. The simulation results show that BS caching can dramatically improve energy efficiency when the content popularity is skewed, the content library size is small, and the backhaul capacity is relatively small. Furthermore, it is confirmed that there exists an optimal BS density which maximizes the energy efficiency of the cache-enabled cellular networks.

\section{Introduction}

Fifth-generation mobile networks (5G) are confronted with the explosive growth of the data traffic and diversified services [1]. In order to handle the ever growing traffic and meet the high quality of service (QoS) requirement of various applications, a large number of new techniques, such as dense cellular networks, full-duplex communications, and massive Multiple-Input Multiple-Output (MIMO), are deployed to boost the data rate of radio access in 5G [2]. However, the limited backhaul hardly copes with the enormous data traffic generated by duplicating downloads of a few popular contents from the core network and becomes the bottleneck of the network performance. Besides, the capacity of storage units has increased substantially while the cost declines consistently in recent years. As a result, cache-enabled cellular networks, in which the popular contents are proactively cached at base stations (BSs) or user equipment (UE), are proposed for content access delay reducing and backhaul traffic releasing recently [3].

In cache-enabled cellular networks, BSs save contents in the cache store according to the specific caching strategy, which makes access protocol, user association, content transmission, and resource allocation different from these of traditional cellular networks. In order to get deep insights into the BS caching, extensive research has been given on the network performance analysis [4-9]. By modeling the network topology with the regular hexagonal grid or the Poisson point process (PPP), various performance metrics in different scenarios had been characterized. The work of [4] derived the hit ratio in a stochastic geometry mode with the limited backhaul. In [5], the author analyzed the successful download probability in two scenarios of the always-on architecture and the dynamic on-off architecture. The work of [6] proposed a collaborative content caching scheme among BSs and compares the average delay per request 
with the noncooperative scenario using queuing theory. The average ergodic rate and the outage probability of a threetier heterogeneous network (HetNet) were studied in [7]. The work of [8] investigated the caching and multicasting in a large-scale cache-enabled HetNet and derives tractable expressions of the successful transmission probability. As for the content caching in UE and sharing the content by deviceto-device (D2D) communications, [9] introduced a proactive caching scheme and quantifies the traffic offloading by cache utility maximization in D2D cellular networks.

In cache-enabled cellular network, contents have been placed at BSs by broadcasting during off-peak times. When the requested files are cached at BSs, users obtain the required contents from local BSs directly and the delivery rate is no longer subject to the limited backhaul capacity. In this case, the system throughput is improved greatly and the power consumption of BS caching should be considered besides the transmission power consumption. When the requested files are not cached at BSs, users need to fetch contents from the core network via the backhaul link. In this case, the limited backhaul capacity not only influences the throughput but also the power consumption of networks. Therefore, it is necessary to analyze the energy efficiency performance of cache-enabled cellular networks with limited backhaul.

1.1. Related Works. Energy efficiency (EE) is a commonly used performance metric in cellular networks [10]. Cacheenabled BSs were proved to be able to improve the energy efficiency significantly compared with the traditional BSs [11]. The work of [12] explored the energy efficiency of cooperative caching in dense cellular networks. A relay caching mechanism for multimedia was designed in [13] to improve the energy efficiency in cellular networks. The work of [14] proposed a social-aware cache information processing approach, in which the social-tie factor (STF) was modeled with the practical data and network performances including throughput, delay, and energy efficiency were derived based on the STF. The work of [15] compared the energy efficiency performance of two scenarios with a macro BS or multiple noncoordinated pico-BSs and confirmed the impact of content popularity and request arrival rate.

Performance analysis was also extended to cache-enabled heterogeneous networks. The work of [16] analyzed the energy efficiency of cache-enabled two-tier HetNet with cochannel/orthogonal channel deployment. The energy efficiency of cache-enabled cooperative dense HetNets was analyzed in [17]. The work of [18] solved the energy-efficient context-aware resource allocation problem with matching algorithm in cache-enabled HetNets. In [19], the caching strategy and base station activation policies of the heterogeneous network were jointly designed to minimize the overall energy consumption. The work of [20] proposed a maximum distance separable (MDS) encoded caching strategy to maximize the energy efficiency of HetNets. The work of [21] presented a cache-enabled software defined HetNet consisting of control-plane (C-plane) and user-plane (Uplane) and analyzed the energy efficiency performance on the assumption that the macro BSs and small BSs cooperate with different cache abilities. The content-related energy efficiency of the cache-enabled D2D network was analyzed in [22].

1.2. Our Contributions. The existing contributions neglect the impact of backhaul link on the energy efficiency of the cacheenabled cellular network. In the realistic networks, the cache capacity of the BSs is limited compared to the enormous content catalog. Since not all contents are cached in BSs, the backhaul link needs to act as a supplement to fetch the content from the core network with the aim of meeting all users demand, which affects the system throughput seriously and generates extra power consumption. In this paper, we investigate the energy efficiency of cache-enabled cellular networks considering the limited backhaul. The main contributions are summarized as follows.

(1) Taking the limited backhaul into account, a cacheenabled cellular network model is developed with the stochastic geometry theory. The network model includes the cellular network model, channel model, cache model, and backhaul model. The backhaul model assumes that the backhaul capacity of each BS is limited. In doing so, the limited backhaul has a great impact on the performance of content delivery in cellular networks, such as throughput and energy efficiency.

(2) Based on the system model, the energy efficiency is theoretically analyzed. Since in cache-enabled cellular networks successful content delivery probability (SCDP) is a basic expression by stochastic geometry analysis, we derived SCDP synthetically considering the wireless channel, BS caching, content popularity distributions, and limited backhaul. Then we derive the exact closed-form expressions of energy efficiency under the constraint of SCDP for one general case and two specific cases of no noise and the mean load approximation.

(3) Simulation results of the energy efficiency of cacheenabled cellular networks with limited backhaul are given. The accuracy of theoretical analysis via Monte Carlo simulations is verified firstly. Then the network parameters, including BS density, cache capacity, content popularity distribution, and the backhaul capacity, are simulated. Simulation results show that $\mathrm{BS}$ caching is able to improve energy efficiency effectively. The performance gain is more obvious under the condition that the content popularity is skewed, the content library size is small, and the backhaul capacity is relatively small. For a fixed cache capacity, there exists an optimal BS density to maximize the energy efficiency of the cache-enabled cellular networks.

The rest of this paper is organized as follows. Section 2 describes the system model. Section 3 derives and analyses the EE of the cache-enabled cellular network. Section 4 validates the theoretical results with simulation analysis. Finally, Section 5 concludes the paper.

\section{System Model}

This section describes the system model; specifically the cellular network model, channel model, cache model, and backhaul model are detailed. 




FIgURE 1: An example of PVT cellular structure.

2.1. Cellular Network Model. Consider a downlink cellular network in this paper. Denoting $x_{i}^{b}, y_{j}^{u}$ as the locations of the $i$ th BS and $j$ th UE, the spatial distributions of BSs and UE obey two independent PPPs $\Phi_{b}=\left\{x_{i}^{b}: i=0,1,2, \ldots\right\}$, $\Phi_{u}=\left\{y_{j}^{u}: j=0,1,2, \ldots\right\}$ in the two-dimensional Euclidean plane, and the intensities are $\lambda_{b}, \lambda_{b}$ accordingly. Each UE is assumed to be associated with the closet BS with the strongest mean received signal strength, leading to the coverage areas which comprise a typical Poisson Voronoi Tessellation (PVT) [27]. Each BS is configured with a cache and stores a certain amount of contents according to the storage capacity. All BSs are connected to the core network via the limited backhaul. An illustration of network topology is shown in Figure 1. According to Palm theory, the statistical properties of UE at any positions coincide with that of a typical UE at a fixed position. Without loss of generality, the analysis is conducted on a typical UE at the origin, namely, the tagged UE $y_{0}^{u}$ in the tagged BS $x_{0}^{b}$.

2.2. Channel Model. The wireless channel gain consists of two types of propagation effect, that is, path loss and Rayleigh fading. The path loss of the tagged UE at a distance of $r$ from the tagged BS is calculated with the power-law model as $r^{-\alpha}$, where $2<\alpha \leq 4$ is the path loss exponent. Rayleigh fading $h$ follows exponential distribution with mean $1, h \sim \exp (1)$. Setting the transmission power of BSs as $P$, thus the received power at the tagged UE can be expressed as $P h r^{-\alpha}$.

Universal frequency reuse is adopted in this paper. All BSs except the tagged BS cause interference to the tagged $\mathrm{UE}$, and the total interference power received is denoted as $I$. Therefore, the signal to interference plus noise ratio (SINR) of the tagged UE can be expressed as

$$
\operatorname{SINR}=\frac{P h z^{-\alpha}}{I+\sigma^{2}}
$$

where $I \triangleq \sum_{i \in \Phi_{b} \mid x_{0}^{b}} P h_{i} z_{i}^{-\alpha}$ indicates the cumulative interference; $\sigma^{2}$ is the additive white Gaussian noise (AWGN).

The system bandwidth is $W$. For tractability, BSs allocate equal spectrum resource to the associated UE. According to the Shannon theory, the downlink rate of the tagged UE is calculated as

$$
R_{a}=\frac{W}{M} \log _{2}(1+\mathrm{SINR}),
$$

where $M$ denotes the total amount of UE served by the tagged BS, namely, the cell load.

2.3. Cache Model. The content catalog contains $N_{f}$ contents, all of which have the same size $F$. For the scenarios of different content sizes, the same analysis is still applicable by splitting the content into chunks of equal size [28]. In this paper, the popularity of the content catalog is known a priori and follows Zipf distribution [29]. We assume that the statistical content popularity distribution changes slowly over time compared with the content delivery in cellular networks. Sorting the content in the descending order of popularity, the popularity of the $k$-ranked content can be described as

$$
P_{N_{f}}(k)=\frac{k^{-\gamma}}{\sum_{g=1}^{N_{f}} g^{-\gamma}},
$$

where $\gamma \geq 0$ is the shape parameter, reflecting the skewness of the popularity distribution. The larger the value of $\gamma$ is, the more uneven the content popularity distribution is.

Due to the limited size of cache space, BSs choose $N_{c}$ most popular contents to cache; cache capacity is $N_{c} F$. Users make content request according to the content popularity. If the requested content is cached in associated BS, the use can directly obtain the content from the local cache. The cache hit ratio can be calculated as

$$
P_{\text {hit }}=\sum_{k=1}^{N_{c}} P_{N_{f}}(k)=\frac{\sum_{k=1}^{N_{c}} k^{-\gamma}}{\sum_{g=1}^{N_{f}} g^{-\gamma}} .
$$

Otherwise, if the requested content is not cached, the user needs the BS to fetch the content from the core network via backhaul link. The cache miss ratio can be calculated as

$$
P_{\text {miss }}=1-P_{\text {hit }}=1-\frac{\sum_{k=1}^{N_{c}} k^{-\gamma}}{\sum_{g=1}^{N_{f}} g^{-\gamma}} .
$$


2.4. Backhaul Model. The backhaul capacity per BS, denoted as $C_{b}$, can be modeled as [4]

$$
C_{b}=\frac{C_{1}}{\lambda_{b}}+C_{2}
$$

where $C_{1}>0, C_{2}>0$ are arbitrary coefficients.

\section{EE of the Cache-Enabled Cellular Network}

This section first derives some auxiliary results containing successful content delivery probability, throughput, and power consumption. Then based on the obtained results, we further analyze the energy efficiency of the backhaulconstrained cache-enabled networks for a general case and two specific cases.

3.1. Successful Content Delivery Probability (SCDP). The successful content delivery probability (SCDP) is defined as the probability that the achievable delivery rate $R$ of the tagged user exceeds the rate demand $R_{0}$ required by the requested content: $\operatorname{Pr}\left(R>R_{0}\right)$. SCDP measuring user's satisfaction with the rate provided with respect to the rate demand is a metric of the quality of service (QoS).

In cache-enabled cellular networks, if cache hit occurs, the user directly gets the requested content from the serving BS and the delivery rate is equal to access delivery rate. SCDP is the probability that the access delivery rate is higher than the rate demand. If cache miss occurs, the user needs the serving BS to fetch the requested content from the core network via backhaul link and forward it to the user via access link. The delivery rate is composed of the access delivery rate and the backhaul delivery rate. SCDP is the probability that both the access delivery rate and the backhaul delivery rate meet the rate demand. To sum up, SCDP is derived as

$$
\begin{aligned}
\operatorname{Pr}\left(R>R_{0}\right)= & P_{\text {hit }} \operatorname{Pr}\left(R_{a} \geq R_{0}\right) \\
& +P_{\text {miss }} \operatorname{Pr}\left(R_{a} \geq R_{0} \& R_{b} \geq R_{0}\right) \\
\stackrel{(a)}{=} & P_{\text {hit }} \operatorname{Pr}\left(R_{a} \geq R_{0}\right) \\
& +\left(1-P_{\text {hit }}\right) \operatorname{Pr}\left(R_{a} \geq R_{0}\right) \operatorname{Pr}\left(R_{b} \geq R_{0}\right) \\
= & \underbrace{\operatorname{Pr}\left(R_{a} \geq R_{0}\right)}_{\text {SADP }} \\
& \cdot(\underbrace{P_{\text {hit }}}_{\text {Cache hit ratio }}+\left(1-P_{\text {hit }}\right) \underbrace{\operatorname{Pr}\left(R_{b} \geq R_{0}\right)}_{\text {SBDP }}) .
\end{aligned}
$$

Equation (a) makes sense due to the fact that the access link and backhaul link are independent of each other. It is shown in (7) that SCDP is mainly determined by three factors, that is, the successful access delivery probability (SADP) $\operatorname{Pr}\left(R_{a} \geq R_{0}\right)$, the successful backhaul delivery probability (SBDP) $\operatorname{Pr}\left(R_{b} \geq R_{0}\right)$, and the cache ratio $P_{\text {hit }}$.
Theorem 1. The successful content delivery probability of the tagged UE in the cache-enabled cellular network is given by the expression

$$
\begin{aligned}
P_{c} & =\sum_{m=1}^{\infty} \int_{0}^{\infty} 2 \pi \lambda_{b} z \\
& \cdot \exp \left\{-\frac{z^{\alpha} \delta \sigma^{2}}{P}-\pi \lambda_{b} z^{2}[H(\delta, \alpha)+1]\right\} d z \\
& \times\left\{P_{h i t}+\left(1-P_{h i t}\right)\left[\sum_{n=0}^{m-1}\left(\begin{array}{c}
m-1 \\
n
\end{array}\right)\right.\right. \\
& \left.\left.\cdot P_{h i t}^{m-n-1}\left(1-P_{h i t}\right)^{n} \min \left(1, \frac{N_{b}}{(n+1)}\right)\right]\right\} \frac{3.5^{3.5}}{m !} \\
& \cdot \frac{\Gamma(m+4.5)}{\Gamma(3.5)}\left(\frac{\lambda_{u}}{\lambda_{b}}\right)^{m}\left(3.5+\frac{\lambda_{u}}{\lambda_{b}}\right)^{-(m+4.5)},
\end{aligned}
$$

where $\delta=2^{m\left(R_{0} / W\right)}-1, H(\delta, \alpha)=(2 \delta /(\alpha-2)){ }_{2} F_{1}(1,1-$ $2 / \alpha ; 2-2 / \alpha ;-\delta)$, and ${ }_{2} F_{1}$ is the hypergeometric function.

Proof. According to the definition of SCDP, we can further deduce the formula

$$
\begin{aligned}
\operatorname{Pr} & \left(R>R_{0}\right) \\
= & \sum_{m=1}^{\infty} \operatorname{Pr}\left(R_{a} \geq R_{0} \mid m\right) \\
& \quad \times\left(P_{\text {hit }}+\left(1-P_{\text {hit }}\right) \operatorname{Pr}\left(R_{b} \geq R_{0} \mid m\right)\right) P_{L}(m),
\end{aligned}
$$

where $P_{L}(m)$ denotes the distribution of the load serviced by the tagged BS [30] and is given by

$$
\begin{aligned}
P_{L} & (M=m+1) \\
& =\frac{3.5^{3.5}}{m !} \frac{\Gamma(m+4.5)}{\Gamma(3.5)}\left(\frac{\lambda_{u}}{\lambda_{b}}\right)^{m}\left(3.5+\frac{\lambda_{u}}{\lambda_{b}}\right)^{-(m+4.5)} .
\end{aligned}
$$

The SADP and SBDP are discussed, respectively, based on the load $m$ of the tagged BS.

(1) Successful Access Delivery Probability (SADP). SADP is the probability that the access delivery rate $R_{w}$ achieved at the tagged UE is higher than the rate demand $R_{0}$ and can be calculated as

$$
\begin{aligned}
P_{a} & =\operatorname{Pr}\left(R_{a} \geq R_{0} \mid m\right) \\
& =\operatorname{Pr}\left(\frac{W}{m} \log (1+\operatorname{SINR}(z)) \geq R_{0}\right) \\
& =\operatorname{Pr}\left(\operatorname{SINR}(z) \geq 2^{m\left(R_{0} / W\right)}-1\right) .
\end{aligned}
$$

Defining $2^{m\left(R_{0} / W\right)}-1=\delta$ as the equivalent SINR threshold, $\operatorname{Pr}(\mathrm{SINR} \geq \delta)$ denotes the coverage probability. As proved in [31], the coverage probability can be expressed as

$$
\begin{aligned}
& \operatorname{Pr}(\operatorname{SINR} \geq \delta)=2 \pi \lambda_{b} \int_{0}^{\infty} z \exp \left(-\frac{z^{\alpha} \delta \sigma^{2}}{P}\right) \\
& \cdot \exp \left(-\pi \lambda_{b}(H(\delta, \alpha)+1) z^{2}\right) d z .
\end{aligned}
$$


SADP is derived as

$$
\begin{aligned}
P_{a} & =2 \pi \lambda_{b} \int_{0}^{\infty} z \exp \left(-\frac{z^{\alpha}\left(2^{m\left(R_{0} / W\right)}-1\right) \sigma^{2}}{P}\right) \\
& \times \exp \left(-\pi \lambda_{b}\left(H\left(\left(2^{m\left(R_{0} / W\right)}-1\right), \alpha\right)+1\right) z^{2}\right) d z
\end{aligned}
$$

(2) Successful Backhaul Delivery Probability (SBDP). Due to the limited backhaul capacity, the maximum number of users supported at the same time by the backhaul link is fixed as $N_{b}=C_{b} / R_{0}$. Denote the number of cache miss users as $N_{\text {miss }}$; if $N_{\text {miss }} \leq N_{b}$, all the cache miss users can be scheduled with the backhaul link. On the contrary, if $N_{\text {miss }}>N_{b}$, the backhaul link fails to support all the cache miss users and will randomly pick $N_{b}$ users. The probability of the backhaul delivery for each user is the same and equal to $N_{\text {miss }} / N_{b}$.

Assuming that the requested content of the tagged user is not cached and there are another $n$ cache miss users associated with the tagged BS, the successful backhaul delivery probability of the tagged UE is given by

$$
\begin{aligned}
P_{b} & =\operatorname{Pr}\left(R_{b} \geq R_{0} \mid m\right) \\
= & \sum_{n=0}^{N_{b}-1}\left(\begin{array}{c}
m-1 \\
n
\end{array}\right) P_{\mathrm{hit}}^{N_{b}-n-1}\left(1-P_{\mathrm{hit}}\right)^{n} \\
& +\sum_{n=N_{b}}^{m-1}\left(\begin{array}{c}
m-1 \\
n
\end{array}\right) P_{\mathrm{hit}}^{m-n-1}\left(1-P_{\mathrm{hit}}\right)^{n} \frac{N_{b}}{(n+1)} \\
= & \sum_{n=0}^{m-1}\left(\begin{array}{c}
m-1 \\
n
\end{array}\right) P_{\mathrm{hit}}^{m-n-1}\left(1-P_{\mathrm{hit}}\right)^{n} \min \left\{1, \frac{N_{b}}{(n+1)}\right\} .
\end{aligned}
$$

Substituting (4), (13), and (14) in (7), Theorem 1 is proved.

Theorem 1 indicates that SCDP mainly depends on three network parameters sets: the first set is related to the access link containing the BS density, transmit power, and path loss parameter; the second set is backhaul capacity, which is associated with backhaul link; the third set is concerned with the cache including the content popularity and cache capacity.

The SCDP expression for general case (8) containing one improper integral of the distance $z$, one infinite summation over the load $m$, and one judgment of the minimum and especial use of lookup tables for ${ }_{2} F_{1}$ is of high computational complexity. In order to analyze the SCDP more conveniently and effectively, two approximate results for the special cases are derived in the following corollaries.

Corollary 2. The successful content delivery probability in interference-limited scenario is simplified as

$$
\begin{aligned}
P_{c}^{\prime} & =\sum_{m=0}^{\infty} \frac{1}{H(\delta, \alpha)+1}\left\{P_{h i t}+\left(1-P_{h i t}\right)\left[\sum_{n=0}^{m-1}\left(\begin{array}{c}
m-1 \\
n
\end{array}\right)\right.\right. \\
& \left.\left.\cdot P_{h i t}^{m-n-1}\left(1-P_{h i t}\right)^{n} \min \left(1, \frac{N_{b}}{(n+1)}\right)\right]\right\} \times \frac{3.5^{3.5}}{m !} \\
& \cdot \frac{\Gamma(m+4.5)}{\Gamma(3.5)}\left(\frac{\lambda_{u}}{\lambda_{b}}\right)^{m}\left(3.5+\frac{\lambda_{u}}{\lambda_{b}}\right)^{-(m+4.5)} .
\end{aligned}
$$

Proof. In interference-limited scenario, the noise power is small with respect to the interference power. Letting $\sigma^{2} \rightarrow$ 0 in (12) and calculating the integral of $z$, the coverage probability neglecting noise can be expressed as

$$
\operatorname{Pr}(\operatorname{SIR} \geq \delta)=\frac{1}{H(\delta, \alpha)+1}
$$

Substituting $\delta=2^{m\left(R_{0} / W\right)}-1$ into (16), we get the SADP in interference-limited scenario as

$$
P_{a}^{\prime}=\frac{1}{H\left(2^{m\left(R_{0} / W\right)}-1, \alpha\right)+1} .
$$

The noise power only has effect on SADP. Multiplying (17) with the unchanged SBDP expression (14), Corollary 2 is proved.

Corollary 3. The successful content delivery probability with the approximation of the mean load in interference-limited scenario is given by

$$
\begin{aligned}
P_{c}^{a v e} & =\frac{1}{H\left(\delta^{\text {ave }}, \alpha\right)+1}\left\{P_{h i t}+\left(1-P_{h i t}\right)\right. \\
\cdot & {\left[\sum_{n=0}^{1.28\left(\lambda_{u} / \lambda_{b}\right)}\left(\begin{array}{c}
1.28 \frac{\lambda_{u}}{\lambda_{b}} \\
n
\end{array}\right) P_{h i t}^{1.28\left(\lambda_{u} / \lambda_{b}\right)-n}\left(1-P_{h i t}\right)^{n}\right.} \\
\cdot & \left.\left.\min \left(1, \frac{N_{b}}{(n+1)}\right)\right]\right\},
\end{aligned}
$$

where $\delta^{\text {ave }}=2^{\left(1+1.28\left(\lambda_{u} / \lambda_{b}\right)\right)\left(R_{0} / W\right)}-1$.

Proof. As proved in [30], the mean load of the tagged BS can be calculated as

$$
\mathbb{E}_{M}=1+1.28 \frac{\lambda_{u}}{\lambda_{b}}
$$

Substituting $1+1.28\left(\lambda_{u} / \lambda_{b}\right)$ within (17) and (14), the SADP and SBDP with the mean load approximation in interferencelimited scenario are derived, respectively, as

$$
\begin{aligned}
P_{a}^{\mathrm{ave}}= & \frac{1}{H\left(2^{\left(1+1.28\left(\lambda_{u} / \lambda_{b}\right)\right)\left(R_{0} / W\right)}-1, \alpha\right)+1}, \\
P_{b}^{\mathrm{ave}}= & \sum_{n=0}^{1.28\left(\lambda_{u} / \lambda_{b}\right)}\left(\begin{array}{c}
1.28 \frac{\lambda_{u}}{\lambda_{b}} \\
n
\end{array}\right) P_{\text {hit }}^{1.28\left(\lambda_{u} / \lambda_{b}\right)-n}\left(1-P_{\text {hit }}\right)^{n} \\
& \times \min \left\{1, \frac{N_{b}}{(n+1)}\right\} .
\end{aligned}
$$

Using an approximation with $\mathbb{E}_{M}\left[P_{c}(m)\right] \approx P_{c}\left(\mathbb{E}_{M}\right)$ and eliminating the summation over $m$, simplified expression (18) is obtained. 
3.2. Energy Efficiency. The throughput defined in this paper is the successful delivery throughput and can be derived as

$$
T=\lambda_{u} \bar{R}_{\text {suc }}
$$

where $\bar{R}_{\text {suc }}$ is the average delivery rate of the typical UE under the premise of the successful content delivery.

In backhaul-constrained cache-enabled cellular networks, if the requested content is cached in the local BS, $\bar{R}_{\text {suc }}$ equals the average successful access delivery rate. If the requested content is not cached and needs to be fetched from the core network, $\bar{R}_{\text {suc }}$ is equivalent to the average successful backhaul delivery rate defined as the minimum required rate $R_{0}$ on the condition that access link and backhaul link are successfully provided. According to the total probability law, the average successful delivery rate $\bar{R}_{\text {suc }}$ can be derived as

$$
\bar{R}_{\text {suc }}=P_{\text {hit }} \bar{R}_{\text {suc }}^{a}+P_{\text {miss }} P_{a} P_{b} R_{0} .
$$

Lemma 4. The average successful access delivery rate in the cache-enabled cellular networks is given by

$$
\begin{aligned}
& \bar{R}_{s u c}^{a}=R_{0}+\int_{R_{0}}^{\infty} \int_{0}^{\infty} \sum_{m=1}^{\infty} 2 \pi \lambda_{b} z \\
& \quad \cdot \exp \left(-\frac{z^{\alpha}\left(\delta_{t}-\delta\right) \sigma^{2}}{P}\right) \\
& \quad \times \exp \left(-\pi \lambda_{b} z^{2}\left(1+H\left(\delta_{t}, \alpha\right)-H(\delta, \alpha)\right)\right) \\
& \quad \cdot P_{L}(m) d z d t,
\end{aligned}
$$

where $\delta_{t}=2^{m(t / W)}-1$ and $P_{L}(m)$ is the load distribution in (10).

Proof. Please refer to the Appendix.

Combining the average successful access delivery rate (24), successful access delivery probability (13), and successful backhaul delivery probability (14) together, we can get the average successful delivery rate in general scenario.

Theorem 5. The average successful delivery rate in the cacheenabled cellular network is given by

$$
\begin{aligned}
& \bar{R}_{s u c}=P_{h i t}\left(R_{0}+\int_{R_{0}}^{\infty} \int_{0}^{\infty} \sum_{m=1}^{\infty} 2 \pi \lambda_{b} z\right. \\
& \cdot \exp \left(-\frac{z^{\alpha}\left(\delta_{t}-\delta\right) \sigma^{2}}{P}\right) \\
& \cdot \exp \left(-\pi \lambda_{b} z^{2}\left(1+H\left(\delta_{t}, \alpha\right)-H(\delta, \alpha)\right)\right) \\
& \left.\cdot P_{L}(m) d z d t\right)+\left(1-P_{h i t}\right) R_{0} \sum_{m=1}^{\infty} P_{a}(m) \\
& \cdot P_{b}(m) P_{L}(m),
\end{aligned}
$$

where $\delta_{t}=2^{m(t / W)}-1$ and $P_{L}(m)$ is the load distribution in (10).
Two special cases are derived to further simplify the average successful delivery rate.

Corollary 6. In interference-limited scenario $\sigma^{2} \rightarrow 0$, the average successful delivery rate is expressed as

$$
\begin{aligned}
\bar{R}_{\text {suc }} & =P_{\text {hit }}\left(R_{0}\right. \\
& \left.+\int_{R_{0}}^{\infty} \sum_{m=1}^{\infty} \frac{1}{1+H\left(\delta_{t}, \alpha\right)-H(\delta, \alpha)} P_{L}(m) d t\right)+(1 \\
& \left.-P_{h i t}\right) R_{0} \sum_{m=1}^{\infty} P_{a}^{\prime}(m) P_{b}(m) P_{L}(m),
\end{aligned}
$$

where $P_{w}^{\prime}(m)$ is the SADP neglecting the noise in (17).

Corollary 7. The average successful delivery rate with the approximation of the mean load in interference-limited scenario is expressed as

$$
\begin{aligned}
\bar{R}_{\text {suc }}^{\text {ave }} & \\
= & P_{\text {hit }}\left(R_{0}+\int_{R_{0}}^{\infty} \frac{1}{1+H\left(\delta_{t}^{\text {ave }}, \alpha\right)-H\left(\delta^{\text {ave }}, \alpha\right)} d t\right) \\
& +\left(1-P_{\text {hit }}\right) R_{0} P_{a}^{\text {ave }} P_{b}^{\text {ave }},
\end{aligned}
$$

where $\delta_{t}^{\text {ave }}=2^{\left(1+1.28\left(\lambda_{u} / \lambda_{b}\right)\right)(t / W)}-1 ; P_{w}^{\text {ave }}$ and $P_{b}^{\text {ave }}$ denote SADP, $S B D P$ with the mean load approximation, respectively, in (20) and (21).

The total power consumed at a BS in cache-enabled cellular network consists of three parts, namely, BSs power consumption, cache power consumption, and backhaul power consumption [24]

$$
\text { Pow }=\lambda_{b}\left(\text { Pow }_{a}+\text { Pow }_{c}+\text { Pow }_{b}\right) .
$$

BS power consumption contains transmit power consumption $P_{t}$ and circuit power consumption $P_{o}$ and is given by

$$
\operatorname{Pow}_{a}=\varepsilon P_{t}+P_{o},
$$

where $\varepsilon$ is the power amplifier efficiency.

Energy-proportional model is used to describe the cache power consumption. In this model, the cache power consumption is proportional to the cache capacity and can be calculated as

$$
\operatorname{Pow}_{c}=\rho N_{c} F,
$$

where $\rho$ denotes the power coefficient of cache hardware.

Backhaul power consumption depends on the successful backhaul delivery throughput at a BS calculated by multiplying the backhaul usage ratio with the backhaul capacity and can be expressed by

$$
\begin{aligned}
& \mathrm{Pow}_{b}=\omega T_{b} \\
& =\omega C_{b} \sum_{m=1}^{\infty} \sum_{n=1}^{m}\left(\begin{array}{l}
m \\
n
\end{array}\right) P_{\mathrm{hit}}^{m-n}\left(1-\mathrm{P}_{\text {hit }}\right)^{n} \min \left\{1, \frac{n}{N_{b}}\right\},
\end{aligned}
$$

where $\omega$ is the power coefficient of the backhaul. 
With the mean load approximation, backhaul power consumption can be simplified as

$$
\begin{aligned}
\operatorname{Pow}_{b}^{\text {ave }}= & \omega C_{b} \sum_{n=1}^{1+1.28\left(\lambda_{u} / \lambda_{b}\right)}\left(\begin{array}{c}
1+1.28 \frac{\lambda_{u}}{\lambda_{b}} \\
n
\end{array}\right) \\
& \times P_{\text {hit }}^{1+1.28\left(\lambda_{u} / \lambda_{b}\right)-n}\left(1-P_{\mathrm{hit}}\right)^{n} \min \left\{1, \frac{n}{N_{b}}\right\} .
\end{aligned}
$$

In conclusion, the total power consumption and the corresponding approximation using the mean load are derived as

$$
\begin{aligned}
\text { Pow } & =\lambda_{b}\left(\text { Pow }_{a}+\text { Pow }_{c}+\text { Pow }_{b}\right), \\
\text { Pow }^{\text {ave }} & =\lambda_{b}\left(\text { Pow }_{a}+\text { Pow }_{c}+\text { Pow }_{b}^{\text {ave }}\right) .
\end{aligned}
$$

According to the definition, we can get the energy efficiency of the cache-enabled cellular networks by dividing the throughput by total power consumption for various scenarios in the sequel.

The energy efficiency for the general case can be calculated with (22), (25), and (33).

$$
\eta_{\mathrm{EE}}=\frac{T}{\text { Pow }}=\frac{\lambda_{u} \bar{R}_{\mathrm{suc}}}{\text { Pow }} .
$$

The energy efficiency in interference-limited scenario can be derived with (22), (26), and (33).

$$
\eta_{\mathrm{EE}}^{\prime}=\frac{T^{\prime}}{\text { Pow }}=\frac{\lambda_{u} \bar{R}_{\mathrm{suc}}^{\prime}}{\text { Pow }} .
$$

The energy efficiency with the approximation of the mean load in interference-limited scenario can be calculated with (22), (27), and (34).

$$
\eta_{\mathrm{EE}}^{\mathrm{ave}}=\frac{T_{\mathrm{ave}}^{\mathrm{ave}}}{\text { Pow }^{\mathrm{ave}}}=\frac{\lambda_{u} \bar{R}_{\mathrm{suc}}^{\mathrm{ave}}}{\text { Pow }^{\text {ave }}} .
$$

\section{Simulation Analysis}

This section presents both the numerical and Monte Carlo simulation results. The scenario of homogeneous cellular network is considered, in which UE and BSs are distributed according to PPP in a circular area with $200 \mathrm{~km}$ radius. The simulation parameters are listed in Table 1 and the results are averaged over 10000 Monte Carlo trials. We validate the analysis in the previous section via Monte Carlo simulations and investigate the impact of various parameters on the system performance containing SCDP and EE.

4.1. SCDP Performance. Figures 2-5 show the performance of SCDP under different system parameters, specifically including the BSs density, the cache capacity, and the backhaul capacity. There are four different curves in each figure. From the figures we can see that the simulation curve matches with the numerical curves well and thus validates the numerical results obtained above. The general result and the no noise result obtained, respectively, from Theorem 1 and Corollary 2
TABLE 1: Simulation parameters.

\begin{tabular}{lc}
\hline Parameter & Value \\
\hline UEs density $\lambda_{u}$ & $0.00024 \mathrm{~m}^{-1}$ \\
Bandwidth $W$ & $20 \mathrm{MHz}$ \\
Path loss exponent $\alpha$ & $4[23]$ \\
Rate threshold $R_{0}$ & $200 \mathrm{Kbps}$ \\
Transmit power $P_{t}$ & $46 \mathrm{dBm}$ \\
Static power consumption $P_{0}[24]$ & $724.6 \mathrm{~W}$ \\
BS power coefficient $\varepsilon[24]$ & 3.22 \\
Content catalog $N_{f}$ & 10000 \\
Content size $F$ & $10 \mathrm{Mbit}$ \\
Caching power coefficient $\rho[25]$ & $6.25 \times 10^{-12} \mathrm{~W} / \mathrm{bit}$ \\
Backhaul capacity coefficient $C_{2}$ & 0 \\
Backhaul power coefficient $\omega[26]$ & $5 \times 10^{-7} \mathrm{~W} / \mathrm{bps}$ \\
\hline
\end{tabular}

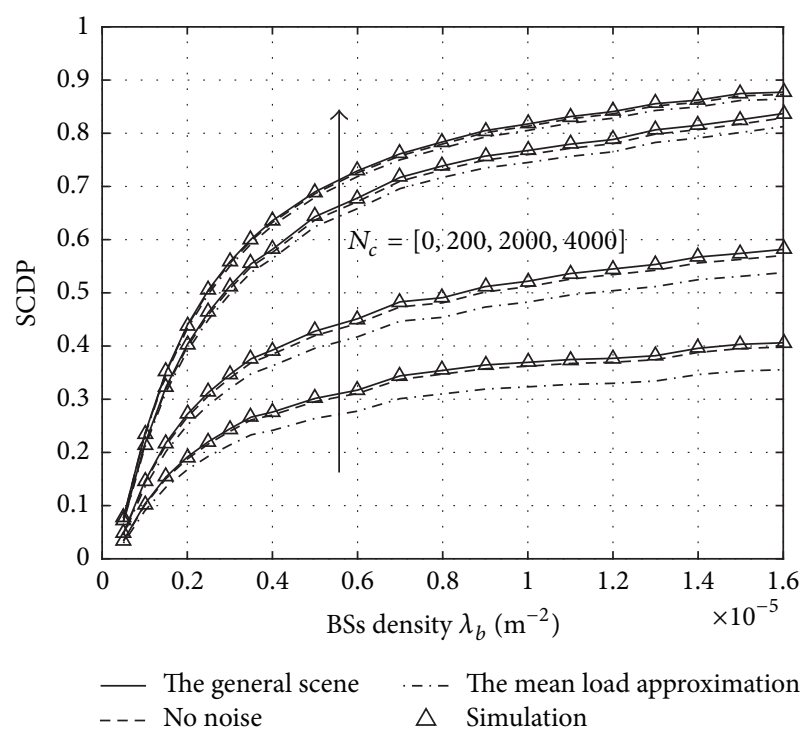

FIGURE 2: SCDP versus BSs density with different cache capacity: $\delta=0.6, N_{f}=10000$, and $C_{1}=24$ bps.

almost overlap, which confirms that noise is not a very important factor in interference-limited cellular networks. The curve shape of the general case and the mean load approximation obtained from Corollary 3 are relatively consistent, but there still exists small gap under some parameter settings. The reason lies in that the approximation error of mean load is aggravated with the operation of summation in (14).

Figure 2 shows the evolution of the SCDP with respect to the BS density for different values of cache capacity. The SCDP increases with the increment of the BS density and gradually converges to a stable value. The main reason is that dense BSs deployment reduces the load at the serving $\mathrm{BS}$ and raises the access delivery rate substantially. However, the increasing BS density aggravates the ICI and hinders the further improvement of the access delivery rate, which eventually results in the saturation of SCDP. Comparing the curves shown for the different cache capacity, BS caching is 


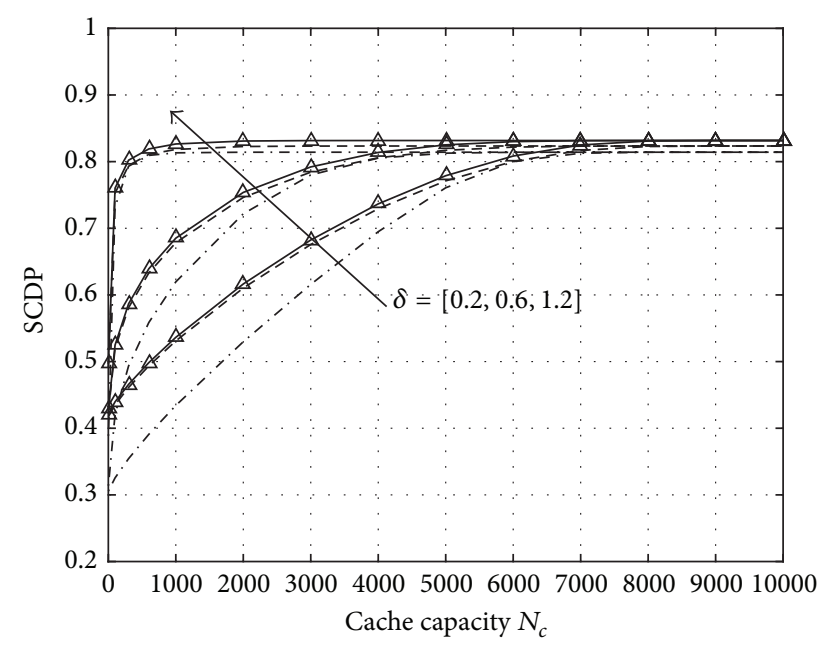



Figure 3: SCDP versus cache capacity with different content popularity parameter: $\lambda_{b}=0.00001 \mathrm{~m}^{-2}, N_{f}=10000$, and $C_{1}=$ 24 bps.



FIGURE 4: SCDP versus cache capacity with different content library size: $\lambda_{b}=0.00001 \mathrm{~m}^{-2}, \delta=0.6$, and $C_{1}=24 \mathrm{bps}$.

proved to be able to increase the SCDP. The larger the cache capacity, the greater the performance gain. The maximum SCDP gain can reach $225 \%$ for $N_{c}=4000$ with respect to no caching.

Figures 3 and 4 investigate the effect of the content popularity distribution in cached enabled cellular networks. Figure 3 shows the SCDP versus cache capacity with different popularity distribution parameter $\delta$. As cache capacity increases, the number of contents stored in the BS grows and the cache hit ratio increases. Since most users obtain the request content from the local BSs and the delivery rate is less affected by the limit of backhaul link, the SCDP is improved.

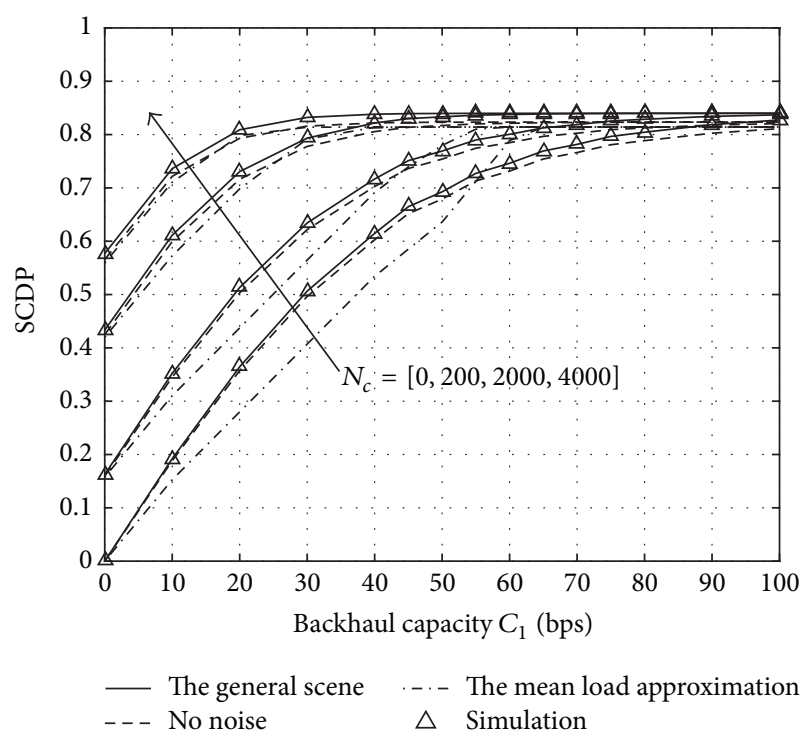

FIGURE 5: SCDP versus backhaul capacity with different cache capacity: $\lambda_{b}=0.00001 \mathrm{~m}^{-2}, \delta=0.6$, and $N_{f}=10000$.

Each curve eventually converges to a point at which BSs caching and backhaul link cooperatively ensure the content availability for each UE and the SCDP is equivalent to the SADP. Furthermore, a large parameter $\delta$, standing for a more concentrated content popularity, results in high cache hit ratio for a fixed cache capacity and further increases the SCDP.

Figure 4 depicts the evolution of SCDP with respect to cache capacity with different content library size $N_{f}$. As shown, the SCDP decreases with the increase of the content library size. If there are a large number of contents, the span of the content popularity distribution is large and the popularity of each content is relatively small for a fixed $\delta$. As a result, the cache hit rate decreases and the SCDP decreases accordingly for a given cache capacity.

The impact of backhaul capacity on the SCDP is shown in Figure 5 for different cache capacity. It can be observed that the increment of backhaul capacity can effectively improve the SCDP due to the increasing SBDP for the cache miss UE. All curves saturate at last, and the larger the cache capacity is, the more quickly the curve saturates, leading to the left shift of the backhaul capacity point that reaches the maximum SCDP.

To sum up, the increment of BSs density, cache capacity, and backhaul capacity can effectively improve the SCDP. Moreover, the performance increases sharply when the content popularity is uneven and the content library size is small.

4.2. EE Performance. Figures 6-9 show the performance of EE with respect to different system parameters containing the BSs density, the cache capacity, and the backhaul capacity. From the figures we can see that the simulation curve and the numerical curve of EE obtained from Theorem 5 and Corollary 6 match well, which gives an effective validation of the numerical results. The general result and the mean load approximation obtained from Corollary 7 are consistent, but 


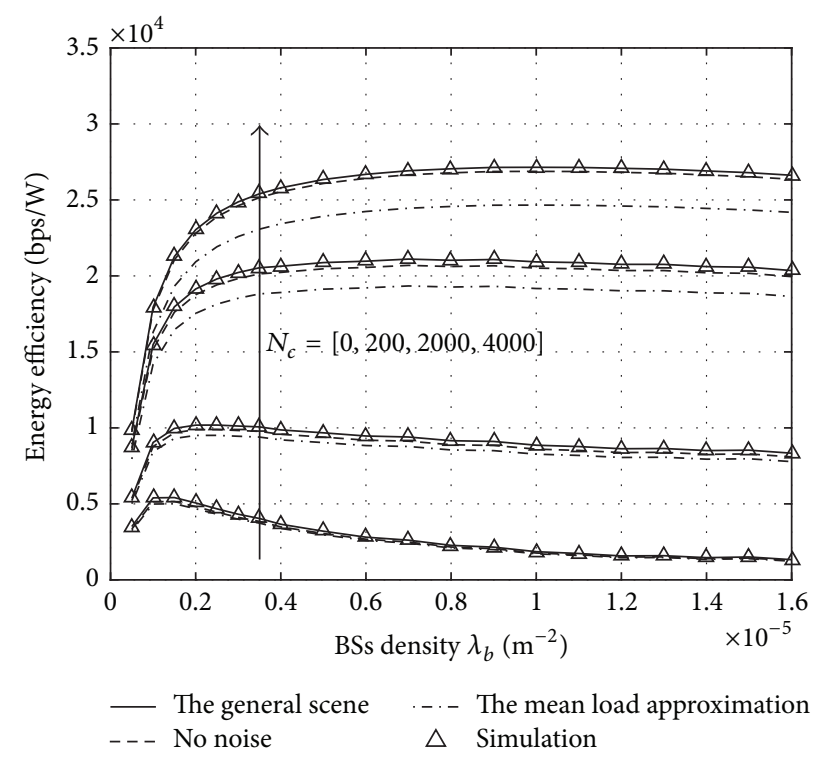

FIGURE 6: EE versus BSs density with different cache capacity: $\delta=$ $0.6, N_{f}=10000$, and $C_{1}=24$ bps.

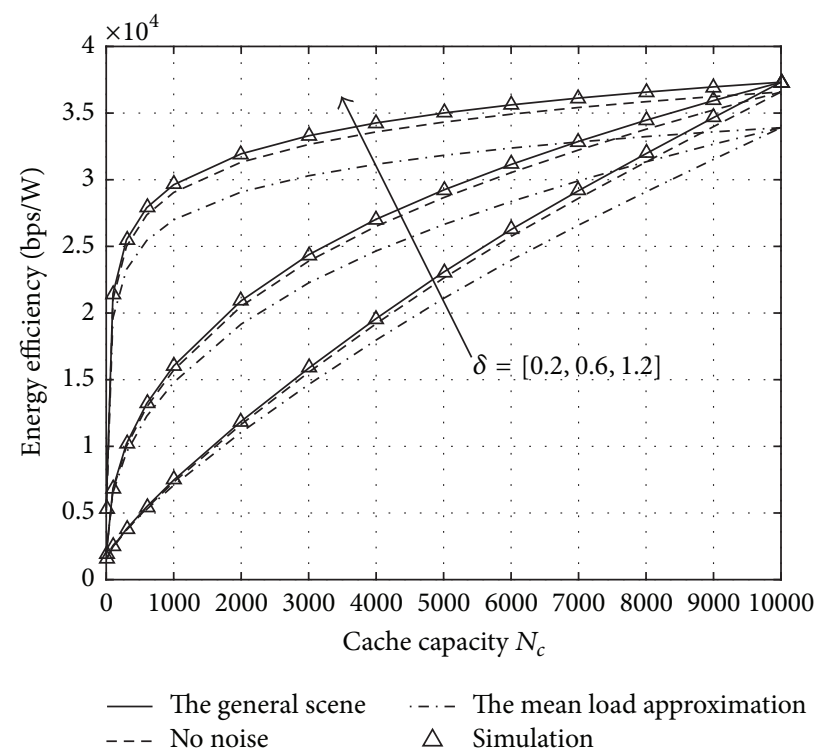

FIGURE 7: EE versus cache capacity with different content popularity parameter: $\lambda_{b}=0.00001 \mathrm{~m}^{-2}, N_{f}=10000$, and $C_{1}=24 \mathrm{bps}$.

there still exists difference due to the fact that the operation of summation in (14) and integral in (24) aggravate the approximation error.

In Figure 6, the EE variation with regard to BSs density for different cache capacity is illustrated. As BSs density increases, the EE first increases benefitting from the improvement of the network throughput and then decreases on account of the large power consumption. There exists an optimal BSs density to maximize the EE. Caching at BSs helps to improve the $\mathrm{EE}$ due to the increment of the delivery rate

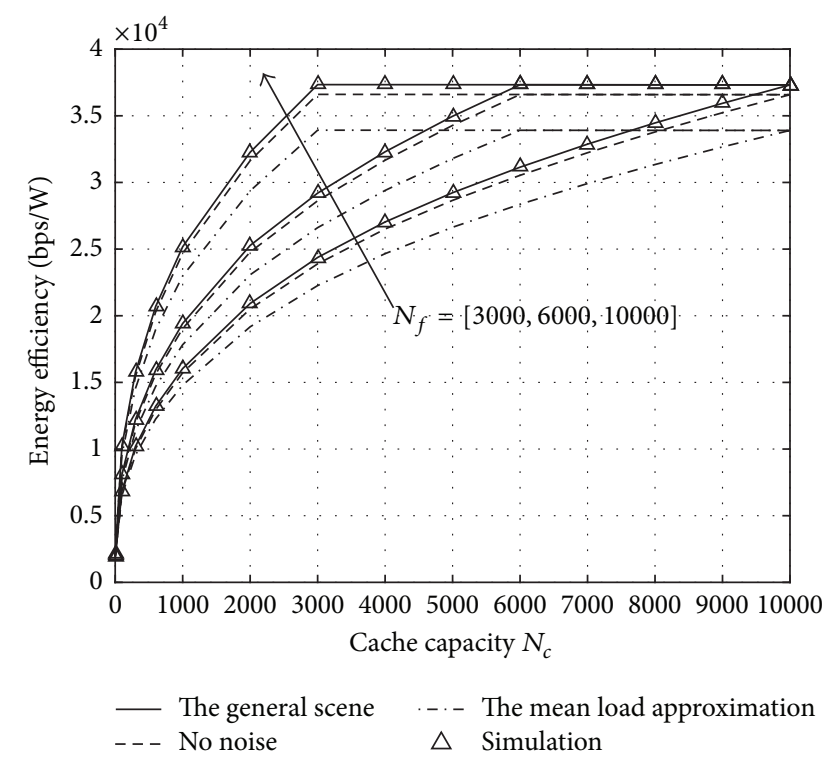

FIGURE 8: EE versus cache capacity with different content library size: $\lambda_{b}=0.00001 \mathrm{~m}^{-2}, \delta=0.6$, and $C_{1}=24 \mathrm{bps}$.

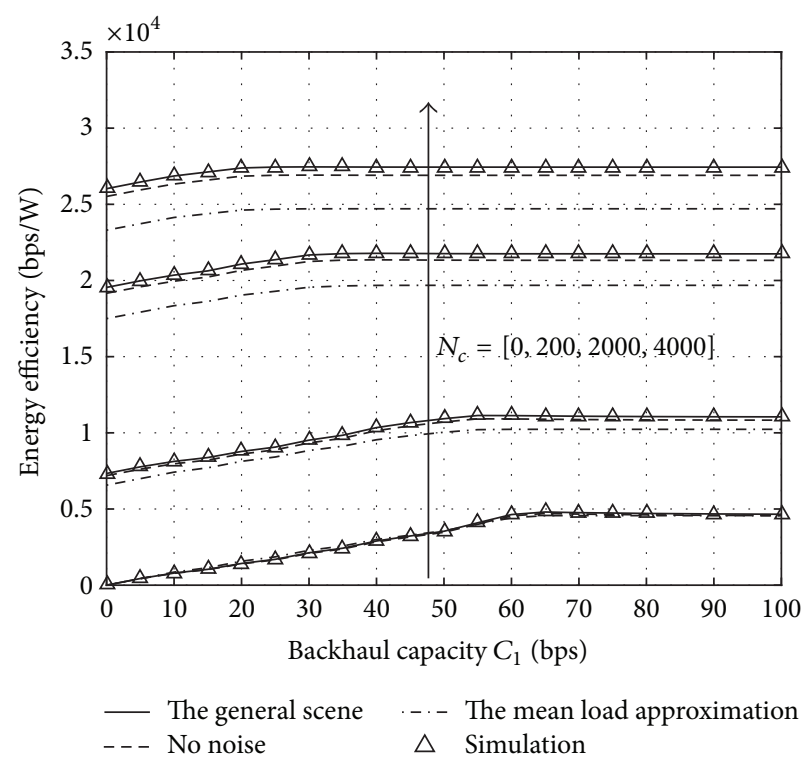

FIGURE 9: EE versus backhaul capacity with different cache capacity: $\lambda_{b}=0.00001 \mathrm{~m}^{-2}, \delta=0.6$, and $N_{f}=10000$.

for the one side and the reduction of the backhaul power consumption for the other side.

Figure 7 shows the influence of cache capacity on the EE with different content popularity distribution parameter $\delta$. With the increase of cache capacity, the performance of EE is improved correspondingly due to the increment of the cache hit ratio until all contents are cached. Moreover, as parameter $\delta$ increases, the EE increases sharply. The reason is that a large amount of UE requests few popular contents; then a cache capacity augmentation can improve the EE more effectively. 
Figure 8 depicts the EE versus cache capacity with different content library size $N_{f}$. As content library shrinks, BS with a fixed cache capacity can store large parts of the contents and the cache hit ratio increases. The EE improves due to the increase of the throughput.

In Figure 9, the relationship between the EE and the backhaul capacity is depicted with different cache capacity. The increasing backhaul capacity increases the throughput of the caching miss UE and improves the EE. EE eventually approaches fixed value, indicating that backhaul capacity is sufficient to support the cache miss UE. BSs caching further enhances the EE. The larger the cache capacity, the smaller the backhaul capacity required to achieve the maximum EE.

In summary, there exists an optimal BS density to maximize the EE for a given cache capacity. The EE can be improved by increasing the cache capacity and the backhaul capacity. The performance gain is large when the content popularity is uneven and the content library size is small.

\section{Conclusion}

In this paper, we analyzed the energy efficiency of cacheenabled cellular networks with the limited backhaul. Using stochastic geometry model, the SCDP is investigated. Based on the auxiliary results of SCDP, we derived the specific expressions of energy efficiency. Simulations study the effect of various factors on system performance and confirm that energy efficiency can be improved dramatically if the content popularity is skewed, the content library size is small, and the backhaul capacity is relatively small. Besides, there exists an optimal BS density to maximize the energy efficiency for a given cache capacity.

\section{Appendix}

Let $f_{Z}(z)=2 \pi \lambda_{b} z e^{-\pi \lambda_{b} z^{2}}$ denote the probability density function (PDF) of the distance $z$ between the tagged user and its serving BS. The average access delivery rate is derived as follows:

$$
\begin{aligned}
\bar{R}_{\text {suc }}^{a} & =E\left(R_{a} \mid R_{a}>R_{0}\right)=\int_{0}^{\infty} P\left(R_{a}>t \mid R_{a}>R_{0}\right) d t=R_{0}+\int_{R_{0}}^{\infty} \frac{P\left(R_{a}>t\right)}{P\left(R_{a}>R_{0}\right)} d t \\
& =R_{0}+\int_{R_{0}}^{\infty} \int_{0}^{\infty} \sum_{m=1}^{\infty} \frac{P\left((W / m) \log _{2}\left(1+P h z^{-\alpha} /\left(I+\sigma^{2}\right)\right) \geq t\right)}{P\left((W / m) \log _{2}\left(1+P h z^{-\alpha} /\left(I+\sigma^{2}\right)\right) \geq R_{0}\right)} \times P_{L}(m) f_{Z}(z) d z d t .
\end{aligned}
$$

$$
\begin{aligned}
& \text { Let } \delta_{t}=2^{m(t / W)}-1 \text { and } \delta=2^{m\left(R_{0} / W\right)}-1 \text {. } \\
& \stackrel{(a)}{=} R_{0}+\int_{R_{0}}^{\infty} \int_{0}^{\infty} \sum_{m=1}^{\infty} \frac{\exp \left(-z^{\alpha} \delta_{t} \sigma^{2} / P\right) E_{I}\left(\exp \left(-z^{\alpha} \delta_{t} I / P\right)\right)}{\exp \left(-z^{\alpha} \delta \sigma^{2} / P\right) E_{I}\left(\exp \left(-z^{\alpha} \delta I / P\right)\right)} \\
& \times P_{L}(m) f_{Z}(z) d z d t \\
& \stackrel{(b)}{=} R_{0} \\
& +\int_{R_{0}}^{\infty} \int_{0}^{\infty} \sum_{m=1}^{\infty} \frac{\exp \left(-z^{\alpha} \delta_{t} \sigma^{2} / P\right) \exp \left(-\pi \lambda_{b} z^{2} H\left(\delta_{t}, \alpha\right)\right)}{\exp \left(-z^{\alpha} \delta \sigma^{2} / P\right) \exp \left(-\pi \lambda_{b} z^{2} H(\delta, \alpha)\right)} \\
& \times P_{L}(m) f_{Z}(z) d z d t=R_{0} \\
& +\int_{R_{0}}^{\infty} \int_{0}^{\infty} \sum_{m=1}^{\infty} 2 \pi \lambda_{b} z \exp \left(-\frac{z^{\alpha}\left(\delta_{t}-\delta\right) \sigma^{2}}{P}\right) \\
& \times \exp \left(-\pi \lambda_{b} z^{2}\left(1+H\left(\delta_{t}, \alpha\right)-H(\delta, \alpha)\right)\right) \\
& \text { - } P_{L}(m) d z d t,
\end{aligned}
$$

where equality ( $a$ ) holds due to $h \sim \exp (1)$; the proof of $(b)$ is similar to Theorem 2 in [31]. The proof is complete.

\section{Conflicts of Interest}

The authors declare that there are no conflicts of interest regarding the publication of this paper.

\section{Acknowledgments}

This paper is supported by National Science and Technology Major Project (no. 2016ZX03001009-003), NSF of China (no. 61502046), the 111 Project (no. B17007), and Director Funds of Beijing Key Laboratory of Network System Architecture and Convergence (2017BKL-NSAC-ZJ-07).

\section{References}

[1] N. Zhao, X. Liu, F. R. Yu, M. Li, and V. C. M. Leung, "Communications, caching, and computing oriented small cell networks with interference alignment," IEEE Communications Magazine, vol. 54, no. 9, pp. 29-35, 2016.

[2] Y. He, Z. Zhang, F. R. Yu et al., "Deep Reinforcement Learningbased Optimization for Cache-enabled Opportunistic Interference Alignment Wireless Networks," IEEE Transactions on Vehicular Technology, 2017.

[3] L. Wang, K.-K. Wong, S. Jin, G. Zheng, and R. W. Heath Jr., "A new look at physical layer security, caching, and wireless energy harvesting for heterogeneous ultra-dense networks," 2017, https://arxiv.org/abs/1705.09647.

[4] E. Bastug, M. Bennis, and M. Debbah, "Cache-enabled small cell networks: Modeling and tradeoffs," in Proceedings of the 2014 11th International Symposium on Wireless Communications Systems, ISWCS 2014, pp. 649-653, Barcelona, Spain, August 2014.

[5] Y. Chen, M. Ding, J. Li, Z. Lin, G. Mao, and L. Hanzo, "Probabilistic small-cell caching: Performance analysis and 
optimization," IEEE Transactions on Vehicular Technology, vol. 66, no. 5, pp. 4341-4354, 2017.

[6] Y. Sun, Z. Chen, and H. Liu, "Delay analysis and optimization in cache-enabled multi-cell cooperative networks," in Proceedings of the 59th IEEE Global Communications Conference, GLOBECOM 2016, Washington, DC, USA, December 2016.

[7] C. Yang, Y. Yao, Z. Chen, and B. Xia, "Analysis on CacheEnabled Wireless Heterogeneous Networks," IEEE Transactions on Wireless Communications, vol. 15, no. 1, pp. 131-145, 2015.

[8] B. Chen and C. Yang, "Energy costs for traffic offloading by cache-enabled D2D communications," in Proceedings of the 2016 IEEE Wireless Communications and Networking Conference, WCNC 2016, Doha, Qatar, April 2016.

[9] T. Zhang, H. Fan, J. Loo, and D. Liu, "User preference aware caching deployment for device-to-device caching networks," IEEE Systems Journal, pp. 1-12, 2017.

[10] J. Tang, D. K. C. So, E. Alsusa, K. A. Hamdi, and A. Shojaeifard, "Resource allocation for energy efficiency optimization in heterogeneous networks," IEEE Journal on Selected Areas in Communications, vol. 33, no. 10, pp. 2104-2117, 2015.

[11] B. Perabathini, E. Bastug, M. Kountouris, M. Debbah, and A. Conte, "Caching at the edge: A green perspective for $5 \mathrm{G}$ networks," in Proceedings of the IEEE International Conference on Communication Workshop, ICCW 2015, pp. 2830-2835, London, UK, June 2015.

[12] J. Liu and S. Sun, "Energy efficiency analysis of dense small cell networks with caching at base stations," in Proceedings of the 2nd IEEE International Conference on Computer and Communications, ICCC 2016, pp. 2944-2948, Chengdu, China, October 2016.

[13] X. Wang, Y. Bao, X. Liu, and Z. Niu, "On the design of relay caching in cellular networks for energy efficiency," in Proceedings of the 2011 IEEE Conference on Computer Communications Workshops, INFOCOM WKSHPS 2011, pp. 259-264, Shanghai, China, April 2011.

[14] J. Zhang, X. Zhang, Z. Yan, Y. Li, W. Wang, and Y. Zhang, "Social-aware cache information processing for $5 \mathrm{G}$ ultra-dense networks," in Proceedings of the 8th International Conference on Wireless Communications and Signal Processing, WCSP 2016, pp. 1-5, October 2016.

[15] D. Liu and C. Yang, "Will caching at base station improve energy efficiency of downlink transmission?" in Proceedings of the 2014 IEEE Global Conference on Signal and Information Processing, GlobalSIP 2014, pp. 173-177, Atlanta, GA, USA, December 2014.

[16] Z. Yan, S. Chen, Y. Ou, and H. Liu, "Energy Efficiency Analysis of Cache-Enabled Two-Tier HetNets under Different Spectrum Deployment Strategies," IEEE Access, vol. 5, pp. 6791-6800, 2017.

[17] J. Liu and S. Sun, "Energy efficiency analysis of cache-enabled cooperative dense small cell networks," IET Communications, vol. 11, no. 4, pp. 477-482, 2017.

[18] Z. Zhou, M. Dong, K. Ota, and Z. Chang, "Energy-efficient context-aware matching for resource allocation in ultra-dense small cells," IEEE Access, vol. 3, pp. 1849-1860, 2015.

[19] K. Poularakis, G. Iosifidis, and L. Tassiulas, "Joint caching and base station activation for green heterogeneous cellular networks," in Proceedings of the IEEE International Conference on Communications, ICC 2015, pp. 3364-3369, London, UK, June 2015.

[20] F. Gabry, V. Bioglio, and I. Land, "On Energy-Efficient Edge Caching in Heterogeneous Networks," IEEE Journal on Selected Areas in Communications, vol. 34, no. 12, pp. 3288-3298, 2016.
[21] J. Zhang, X. Zhang, and W. Wang, "Cache-Enabled Software Defined Heterogeneous Networks for Green and Flexible 5G Networks," IEEE Access, vol. 4, pp. 3591-3604, 2016.

[22] Y. Long, Y. Cai, D. Wu, and L. Qiao, "Content-related energy efficiency analysis in cache-enabled device-to-device network," in Proceedings of the 8th International Conference on Wireless Communications and Signal Processing, WCSP 2016, Yangzhou, China, October 2016.

[23] L. Fan, R. Zhao, F.-K. Gong, N. Yang, and G. K. Karagiannidis, "Secure Multiple Amplify-and-Forward Relaying over Correlated Fading Channels," IEEE Transactions on Communications, vol. 65, no. 7, pp. 2811-2820, 2017.

[24] G. Auer, V. Giannini, C. Desset et al., "How much energy is needed to run a wireless network?" IEEE Wireless Communications Magazine, vol. 18, no. 5, pp. 40-49, 2011.

[25] N. Choi, K. Guan, D. C. Kilper, and G. Atkinson, "In-network caching effect on optimal energy consumption in contentcentric networking," in Proceedings of the IEEE International Conference on Communications (ICC '12), pp. 2889-2894, Ottawa, Canada, June 2012.

[26] A. J. Fehske, P. Marsch, and G. P. Fettweis, "Bit per joule efficiency of cooperating base stations in cellular networks," in Proceedings of the 2010 IEEE Globecom Workshops, GC'10, pp. 1406-1411, USA, December 2010.

[27] D. Stoyan, W. S. Kendall, and J. Mecke, Stochastic Geometry and Its Applications, Wiley, 2nd edition, 1996.

[28] X. Xu and M. Tao, "Modeling, analysis, and optimization of coded caching in small-cell networks," IEEE Transactions on Communications, vol. 65, no. 8, pp. 3415-3428, 2017.

[29] N. Golrezaei, K. Shanmugam, A. G. Dimakis, A. F. Molisch, and G. Caire, "FemtoCaching: Wireless video content delivery through distributed caching helpers," in Proceedings of the IEEE Conference on Computer Communications, INFOCOM 2012, pp. 1107-1115, usa, March 2012.

[30] H. S. Dhillon, R. K. Ganti, F. Baccelli, and J. G. Andrews, "Modeling and analysis of K-tier downlink heterogeneous cellular networks," IEEE Journal on Selected Areas in Communications, vol. 30, no. 3, pp. 550-560, 2012.

[31] J. G. Andrews, F. Baccelli, and R. K. Ganti, "A tractable approach to coverage and rate in cellular networks," IEEE Transactions on Communications, vol. 59, no. 11, pp. 3122-3134, 2011. 


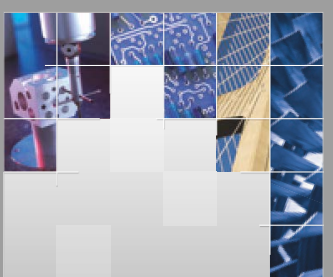

\section{Enfincering}
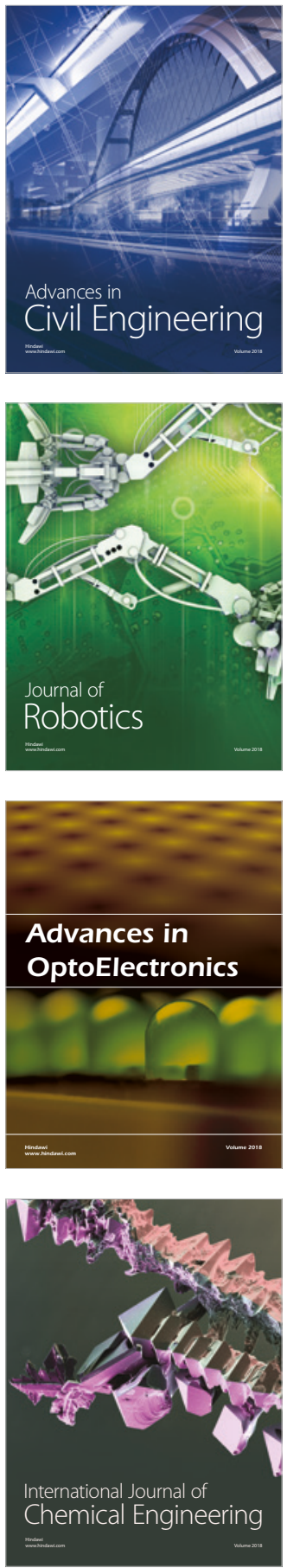

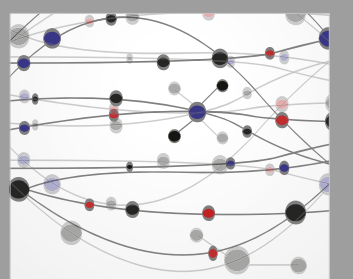

\section{Rotating \\ Machinery}

The Scientific World Journal



Submit your manuscripts at

www.hindawi.com

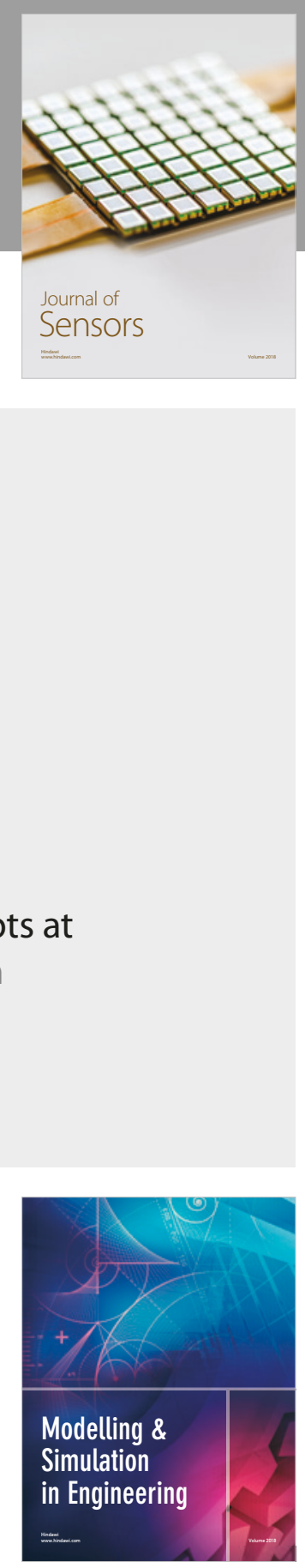

\section{Advances \\ Multimedia}
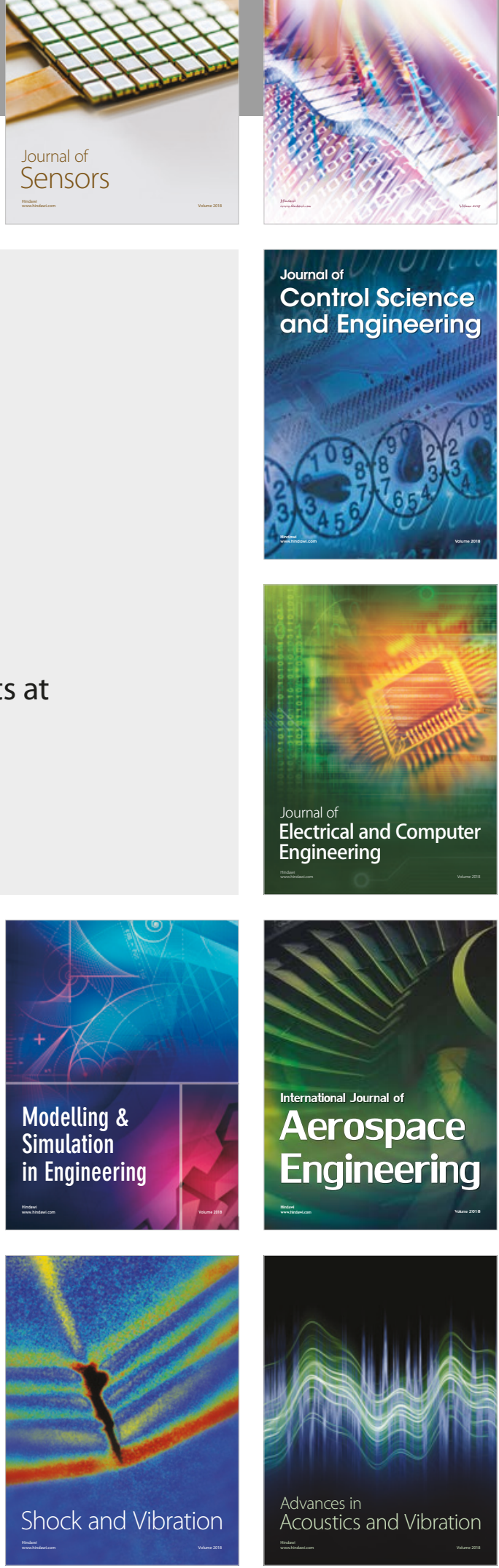